\title{
Behavioral heat-stress compensation in a cold-adapted ungulate: forage-mediated responses to warming Alpine summers
}

\author{
Paola Semenzato ${ }^{1}$, Francesca Cagnacci ${ }^{2}$, Emanuele Eccel $^{2}$, Federico Ossi $^{3}$, Mark Hewison $^{4}$, \\ Nicolas Morellet ${ }^{4}$, Enrico Sturaro ${ }^{5}$, and Maurizio Ramanzin ${ }^{5}$ \\ ${ }^{1}$ University of Padova - Agripolis Campus \\ ${ }^{2}$ Fondazione Edmund Mach Centro Ricerca e Innovazione \\ ${ }^{3}$ University of Trento \\ ${ }^{4}$ CEFS-INRA \\ ${ }^{5}$ University of Padova
}

September 28, 2020

\begin{abstract}
Alpine large herbivores have developed physiological and behavioral mechanisms to cope with fluctuations in climate and resource availability, but climate warming might induce behavioral maladaptation. We verified this hypothesis in female Alpine ibex (Capra ibex) by modelling seasonal and daily movement and activity patterns in function of temperature and vegetation productivity, based on bio-logging data and climate change projections. In late spring, ibex moved upslope, tracking the greenwave in plant phenology. Ibex sharply decreased diel activity above a threshold mean daily temperature of $13^{\circ} \mathrm{C}$, indicating thermal stress, but compensating behaviorally by foraging earlier at dawn, and later at dusk, and by moving upslope higher than on cooler days. This temperature threshold will be exceeded more than three times as often under climate change projections. In such scenarios, the imperative requirement for thermal shelter may force Alpine ibex towards topographic edges, impacting individual performance and population distribution of this emblematic mountain ungulate.
\end{abstract}

\section{Statement of the authorship:}

FC and MR conceived and designed the study. ES, MR, and PS organized and managed animal marking. PS collected and managed the bio-telemetry and observational data. EE processed climate warming projections. PS, FC and MR designed statistical analyses. PS analyzed the data. PS and FC wrote the first draft of the manuscript, with comments from NM, AJMH, FO and MR. All co-authors revised further versions of the manuscript. MR competitively obtained funds to support the study, with contributions from FC.

Data accessibility statement:

Data are currently stored in the Euroungulates database (www.euromammals.org) and access can be provided after $\log$ in. Dataset used for analysis can be further stored on a d.o.i. repository upon acceptance of the manuscript.

\section{Article title:}

Behavioral heat-stress compensation in a cold-adapted ungulate: forage-mediated responses to warming Alpine summers 


\section{Authors:}

Paola Semenzato§

Department of Agronomy Food Natural Resources Animals and Environment (DAFNAE), University of Padova. Viale dell'Università 16, 35020 Legnaro (PD), Italy

paola.semenzato4@gmail.com

Francesca Cagnacci*

Biodiversity and Molecular Ecology Department, Research and Innovation Centre, Fondazione Edmund Mach, Via E. Mach 1, 38010 San Michele all' Adige, Trentino, Italy

francesca.cagnacci@fmach.it

Emanuele Eccel

Research and Innovation Centre, Fondazione Edmund Mach, Via E. Mach 1, 38010 San Michele all' Adige, Trentino, Italy

emanuele.eccel@fmach.it

Federico Ossi

Centre for Agriculture, Food and Environment, University of Trento and

Biodiversity and Molecular Ecology Department, Research and Innovation Centre, Fondazione Edmund Mach, Via E. Mach 1, 38010 San Michele all' Adige, Trentino, Italy

federico.ossi@gmail.com

A. J. Mark Hewison

CEFS, Université de Toulouse, INRA, Castanet Tolosan, France

Mark.Hewison@inra.fr

Nicolas Morellet

CEFS, Université de Toulouse, INRA, BP 52627 - 31326 Castanet-Tolosan, France

Nicolas.Morellet@inra.fr

Enrico Sturaro

Department of Agronomy Food Natural Resources Animals and Environment (DAFNAE), University of Padova. Viale dell'Università 16, 35020 Legnaro (PD), Italy

enrico.sturaro@unipd.it

Maurizio Ramanzin*

Department of Agronomy Food Natural Resources Animals and Environment (DAFNAE), University of Padova, Viale dell'Università 16, 35020 Legnaro (PD), Italy

maurizio.ramanzin@unipd.it

* Maurizio Ramanzin and Francesca Cagnacci are co-senior authors.

$\S$ To whom the correspondence should be addressed.

Running title:

Climate and behavior in Alpine Ibex 
Keywords:

Climate change, Alpine ibex, Capra ibex, foraging, thermoregulation, behavioral responses, altitudinal migration, activity budget, GPS telemetry, accelerometer.

Type of article:

Letters

Number of words:

Abstract: 151 words

Main text: 4937 words

Number of references: 77

6 figures, 0 tables, 0 text boxes

Corresponding author:

Paola Semenzato, Department of Agronomy Food Natural Resources Animals and Environment (DAFNAE), University of Padova. Viale dell'Università 16, 35020 Legnaro (PD), Italy

tel. +390498272664

paola.semenzato4@gmail.com

\section{Hosted file}

Ibex_heat-stress_compensation_ELEsemenzato.pdf available at https://authorea.com/users/ 362550/articles/483661-behavioral-heat-stress-compensation-in-a-cold-adapted-ungulateforage-mediated-responses-to-warming-alpine-summers

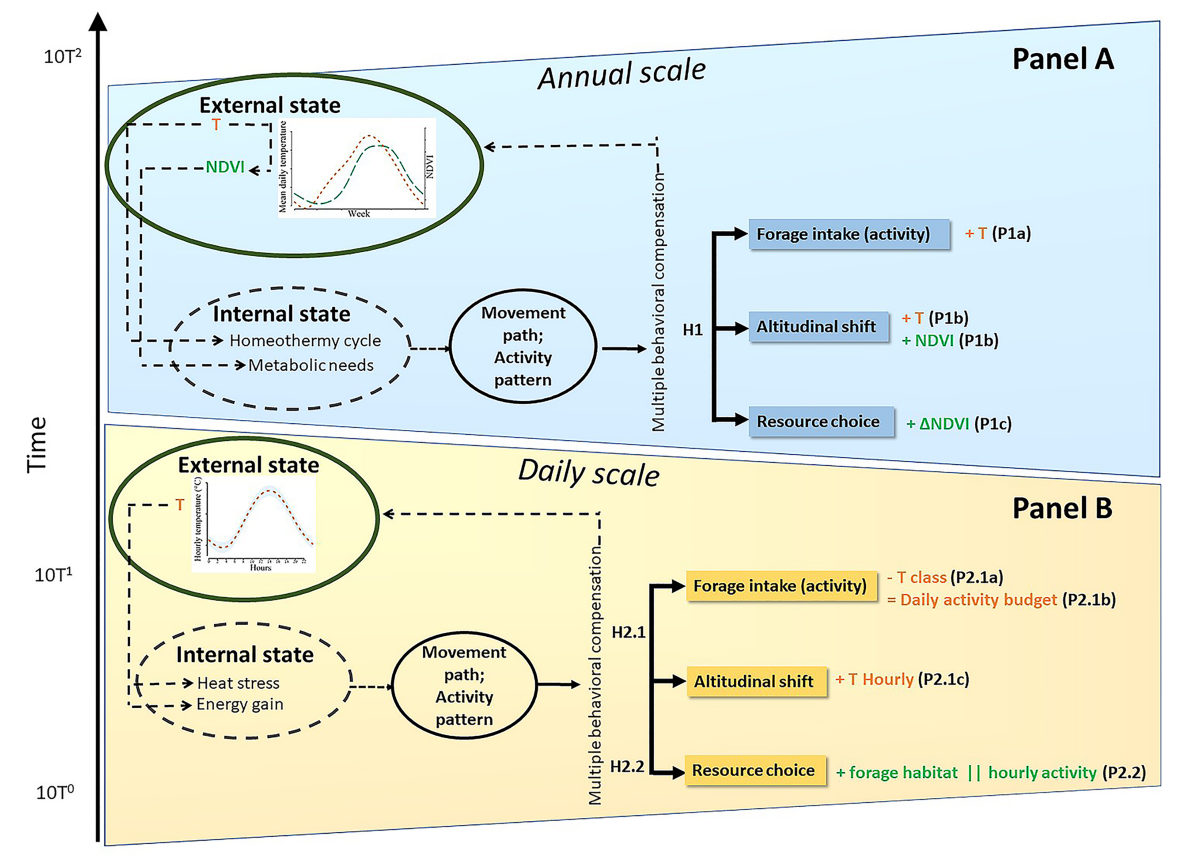



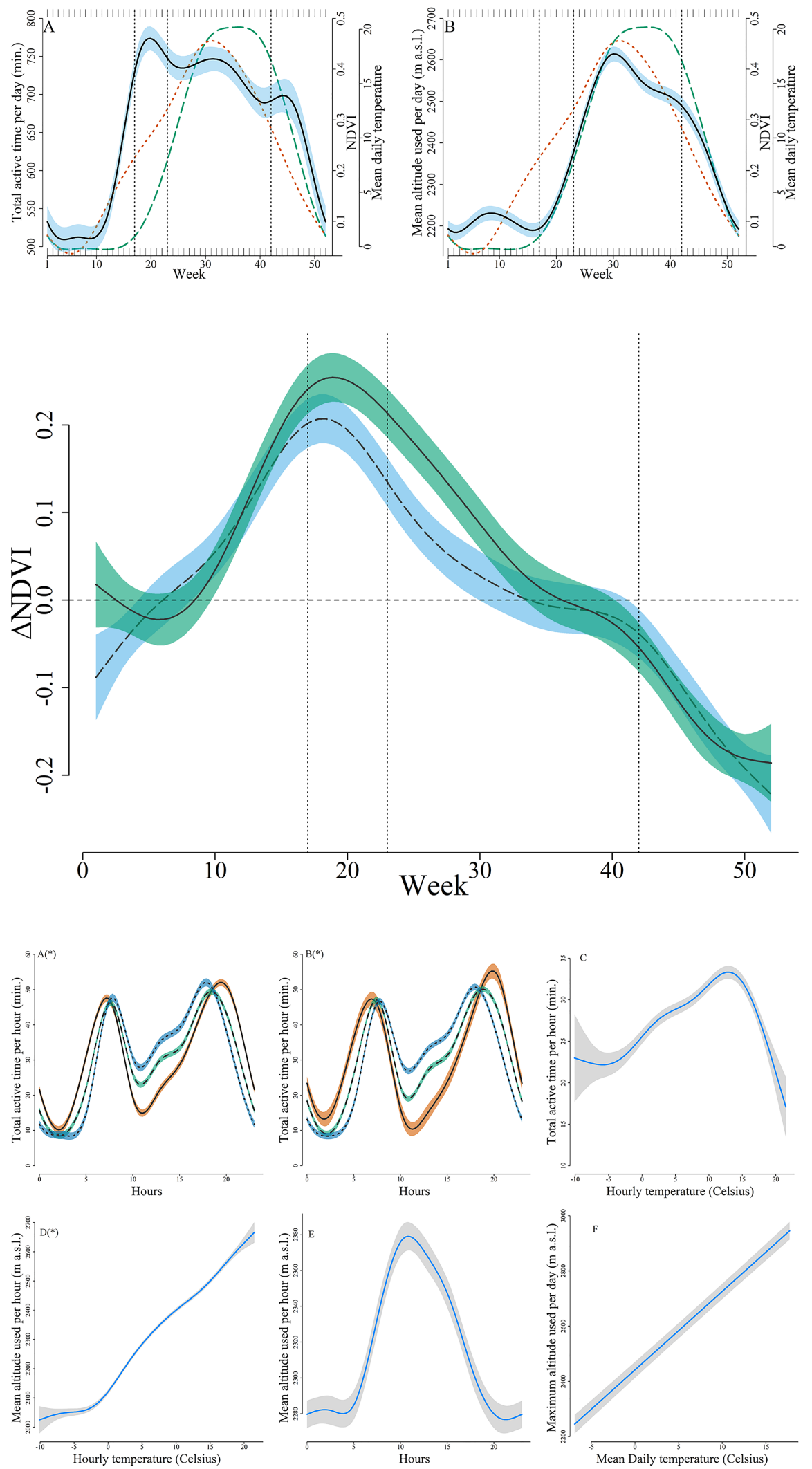

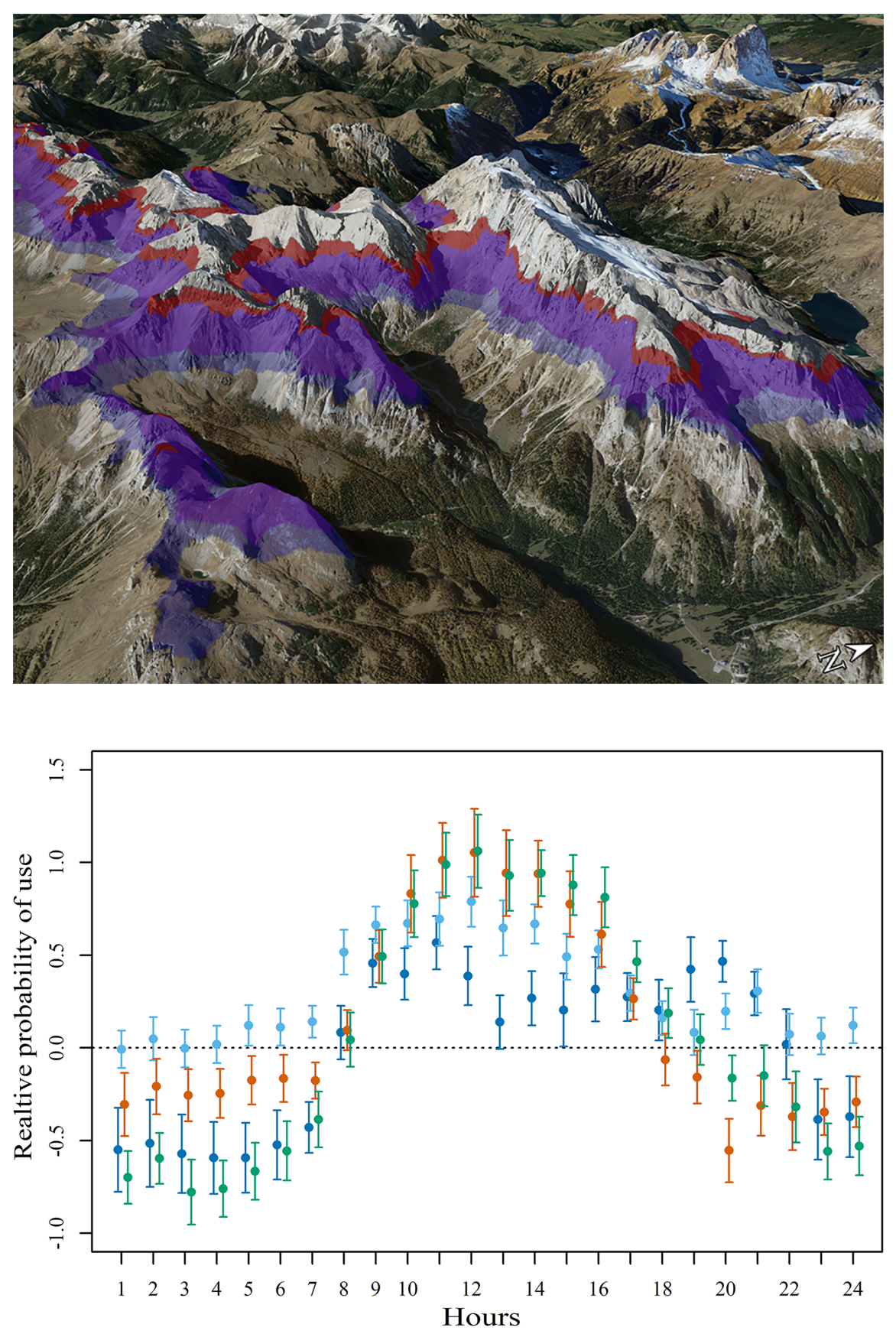